\title{
microRNA-145 inhibits osteosarcoma cell proliferation and invasion by targeting ROCK1
}

\author{
PENGFEI LEI, JIE XIE, LONG WANG, XUCHENG YANG, ZIXUN DAI and YIHE HU \\ Department of Orthopedics, Xiangya Hospital of Central South University, Changsha, Hunan 410008, P.R. China
}

Received September 4, 2013; Accepted March 12, 2014

DOI: $10.3892 / \mathrm{mmr} .2014 .2195$

\begin{abstract}
Osteosarcoma (OS), a malignant mesenchymal sarcoma, is the most frequent primary bone tumor, with a peak incidence in young children and adolescents. The downregulation of microRNA-145 (miRNA/miR-145) has previously been identified to be associated with the aggressiveness and metastasis of OS. However, the detailed regulatory mechanism by which miR-145 inhibits OS remains largely unknown. The present study demonstrated that miR-145 was significantly downregulated in OS tissues and KHOS and U2OS cell lines. Rho-associated protein kinase 1 (ROCK1), a key regulator of actin cytoskeleton reorganization, was identified as a novel target of miR-145. Ectopic expression of miR-145 notably suppressed the protein expression of ROCK1 without affecting its mRNA level. Furthermore, the expression of ROCK 1 was significantly increased in the OS tissues and in the KHOS and U2OS cells. It was further demonstrated that the overexpression of miR-145 downregulated KHOS and U2OS cell proliferation and invasion, which was reversed by restoration of ROCK1. To the best of our knowledge, the present study demonstrates for the first time that, as a tumor suppressor, miRNA-145 inhibits OS cell proliferation and invasion, at least in part by directly targeting ROCK1. These results indicate that miR-145 may be a potential candidate for the diagnosis and treatment of OS.

\section{Introduction}

Osteosarcoma (OS) mainly arises from the metaphysis of the long bones of adolescents and young adults. OS is the most common primary malignant tumor and is associated with high morbidity (1). Despite wide tumor excision combining multi-agent chemotherapy and radiotherapy, the five-year survival rate of patients with recurrent or metastatic OS remains at $\sim 30 \%$ (2). Although recent studies have focused on

Correspondence to: Professor Yihe Hu, Department of Orthopedics, Xiangya Hospital of Central South University, 87 Xiangya Road, Changsha, Hunan 410008, P.R. China

E-mail: csuhuyihe@163.com
\end{abstract}

Key words: osteosarcoma, Rho-associated protein kinase 1 , microRNA-145, proliferation, invasion the molecular pathogenesis of OS (3), its detailed molecular mechanisms have not been fully elucidated. As a result, the identification of novel molecular candidates and/or targets is crucial for the development of effective therapeutic strategies to improve the prognosis of OS.

microRNAs (miRNA/miRs) are small non-coding RNA molecules with 18-25 nucleotides, which mainly negatively regulate gene expression by suppressing translation via binding to the $3^{\prime}$ untranslated region (3'UTR) of their target genes (4). Previously, miRNAs have been demonstrated to have crucial roles in various physiological and pathological processes, including the development and progression of malignant tumors (5). In fact, it has been demonstrated that miRNAs function as oncogenes or tumor suppressors in cellular proliferation, apoptosis, differentiation, migration and invasion in various cancer cells $(6,7)$. Despite indications that several miRNAs, including miR-20a, miR-199a-3p, miR-143 and so forth, are involved in the development and progression of OS (8-10), their exact role remains largely unknown. Previously, miR-145 was identified as a tumor-suppressive miRNA in multiple types of cancers, including lung, glioma, ovarian, colon, gastric, bladder, prostate and breast cancer, as well as in OS (11-17). However, the detailed regulatory mechanism underlying the effects of miR-145 in OS cells remains unclear.

Rho-associated protein kinase 1 (ROCK1) is a serine/threonine protein kinase that has been demonstrated to have a critical role in regulating the actin cytoskeleton (18). Through phosphorylation of downstream substrates, ROCK1 is able to promote actin filament stabilization and actin-myosin contractility (19). Recently, several studies demonstrated that the expression of ROCK1 was increased in several types of malignant tumors (20). Notably, its upregulation was correlated with the poor prognosis of patients with OS (21). Accordingly, ROCK1 may become a promising therapeutic target for OS, and further studies on its actions in OS are consequently urgently required.

This study aimed to investigate the role of miR-145 in the regulation of OS in vitro as well as the underlying molecular mechanisms.

\section{Materials and methods}

Reagents and materials. RPMI-1640, fetal bovine serum (FBS), TRIzol reagent, the TaqMan MicroRNA Assay kit, 
miR-145 mimics and Lipofectamine 2000 were all purchased from Invitrogen Life Technologies (Carlsbad, CA, USA). The PrimeScript RT Reagent kit and SYBR Premix Ex Taq II were purchased from Takara (Dalian, Liaoning, China). The miRNeasy Mini kit was obtained from Qiagen (Valencia, CA, USA). Protein assay reagents were purchased from Bio-Rad (Hercules, CA, USA) and the QuikChange Site-Directed Mutagenesis kit was purchased from Stratagene (La Jolla, CA, USA). The PsiCHECK2 vector and Dual-Luciferase Reporter Assay system were obtained from Promega Corporation (Madison, WI, USA), while the pcDNA3.1 (+)-ROCK1 plasmid was purchased from Supbiology (Changsha, Hunan, China). Mouse anti-ROCK1 monoclonal antibody, mouse anti- $\beta$-actin monoclonal antibody and goat anti-mouse secondary antibody were purchased from Abcam (Cambridge, UK). The Cell Invasion Assay kit was obtained from Merck Millipore (Darmstadt, Germany).

Tissue specimen collection. The present study was approved by the Ethics Committee of Xiangya Hospital of Central South University (Changsha, Hunan, China). Written informed consent was obtained from each patient. A total of 18 primary OS samples and their matched non-cancerous bone tissue samples were collected from patients at the Department of Orthopedics, Xiangya Hospital of Central South University between March 2011 and March 2013. None of the patients had received blood transfusions, radiotherapy or chemotherapy prior to surgery. All samples were immediately snap-frozen in liquid nitrogen following surgical removal and stored at $-80^{\circ} \mathrm{C}$ until use.

Cell culture. The human OS cell lines, KHOS and U2OS, were obtained from the American Type Culture Collection (ATCC; Rockville, MD, USA). The cells were cultured in RPMI-1640 with $10 \% \mathrm{FBS}, 100 \mathrm{U} / \mathrm{ml}$ penicillin and $100 \mathrm{mg} / \mathrm{ml}$ streptomycin in a humidified atmosphere containing $5 \% \mathrm{CO}_{2}$ at $37^{\circ} \mathrm{C}$.

RNA extraction and quantitative (q)PCR. For the mRNA expression assay, total RNA was extracted from the tissues and cells using TRIzol in accordance with the manufacturer's instructions. Reverse transcription PCR was performed with the PrimeScript RT reagent kit. qPCR was performed using SYBR Premix Ex Taq II. The ROCK1-specific primer sequences were as follows: 5'-GGTGGTCGGTTGGGGTAT TTT-3' (forward) and 5'-CGCCCTAACCTCACTTCCC-3' (reverse). $\beta$-actin was used as an endogenous control and its primer sequences were as follows: 5'-CTCCATCCTGGCCT CGCTGT-3' (forward) and 5'-GCTGTCACCTTCACCGTT CC-3' (reverse). For the miRNA expression assay, miRNAs were isolated by the miRNeasy Mini kit. Following this, the TaqMan MicroRNA Assays kit was used to determine the miRNA expression on a 7500 Fast Real Time PCR system (Applied Biosystems, Carlsbad, CA, USA). The universal small nuclear RNA, U6, was used as an endogenous control. For each sample, independent experiments were repeated three times. The relative expression levels of mRNA and miRNA were analyzed by use of the $2^{-\Delta \Delta \mathrm{Ct}}$ method.

Transfection. For transfection, $1 \times 10^{5}$ cells were harvested and seeded in a 24-well plate, and cultured for $24 \mathrm{~h}$. Prior to transfection, the media was replaced to become serum free. Lipofectamine 2000 was used to transfect the miRNA or pcDNA3.1(+)-ROCK1 plasmid into the cells, according to the manufacturer's instructions. At $6 \mathrm{~h}$ post-transfection, the transfection medium was replaced by complete medium. The cells were then cultured in a humidified atmosphere containing $5 \%$ $\mathrm{CO}_{2}$ at $37^{\circ} \mathrm{C}$ for $48 \mathrm{~h}$.

Luciferase reporter assay. The 3'-UTR of ROCK1 containing the miR-145 binding site was cloned into the psiCHECK2 luciferase reporter vector using the following primers: 5'-CGC GGCCGCTAGTCTGTGGAATCGTGTGGGAT-3' (forward) and 5'-ATCCCACACGATTCCACAGACTAGCGGCCGCGA GCT-3'. The mutant 3'-UTR of ROCK1 was generated using a QuikChange Site-Directed Mutagenesis kit. This mutant 3'-UTR of ROCK1 had a substitution of three nucleotides (UGG to GTT) within the seed region of the miR-145 binding site. The miR-145 mimic was then co-transfected with the psiCHECK2 vector inserted, with the wild-type or mutant-type 3'UTR of ROCK1, into the KHOS and U2OS cell lines, respectively, by Lipofectamine 2000 according to the manufacturer's instructions. At $48 \mathrm{~h}$ post-transfection, the luciferase activity for each sample was determined by the Dual-Luciferase Reporter Assay system according to the manufacturer's instructions. All experiments were performed in triplicate. Renilla luciferase was used for normalization.

Western blotting. The tissues and cells were solubilized in cold radioimmunoprecipitation assay lysis buffer. The concentration of protein lysate was determined by protein assay reagents. Following this, the protein was separated with $12 \%$ SDS-PAGE. The protein from each line was then transferred to a PVDF membrane, which was blocked in 5\% skimmed dried milk in phosphate-buffered saline (PBS) at $4^{\circ} \mathrm{C}$ overnight. According to the manufacturer's instructions, the membrane was incubated at room temperature for $3 \mathrm{~h}$ with mouse anti-ROCK1 monoclonal antibody (1:400) or mouse anti- $\beta$-actin monoclonal antibody (1:200), respectively, and then with goat anti-mouse secondary antibody $(1: 20,000)$ for $1 \mathrm{~h}$. An enhanced chemiluminescence reagent was used to detect the signals on the membranes. The data were analyzed by densitometry using Image-Pro plus software 6.0 (Media Cybernetics, Rockville, MD, USA) and normalized to $\beta$-actin expression.

Cell proliferation assay. A cell proliferation assay was performed with MTT (Sigma-Aldrich, St. Louis, MO, USA), according to the manufacturer's instructions. Briefly, $1 \times 10^{4}$ cells/well were plated in a 96 -well plate. The plates were incubated for $24 \mathrm{~h}$ in a humidified atmosphere containing $5 \%$ $\mathrm{CO}_{2}$ at $37^{\circ} \mathrm{C}$. Following this, $50 \mu \mathrm{l} \mathrm{MTT}(5 \mathrm{mg} / \mathrm{ml})$ in PBS was added and incubated for $4 \mathrm{~h}$ in a humidified atmosphere containing $5 \% \mathrm{CO}_{2}$ at $37^{\circ} \mathrm{C}$. Next, $150 \mu \mathrm{l}$ DMSO was added following removal of the supernatant. A microplate reader (Bio-Rad) was used to determine the absorbance at $570 \mathrm{~nm}$. Each assay was performed in triplicate wells and repeated three times.

Invasion assay. According to the manufacturer's instructions, the cell suspension containing $5 \times 10^{5}$ cells $/ \mathrm{ml}$ was prepared in 
A

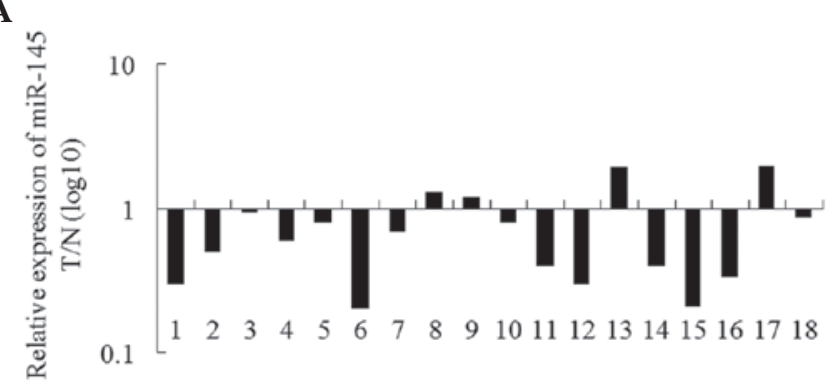

B

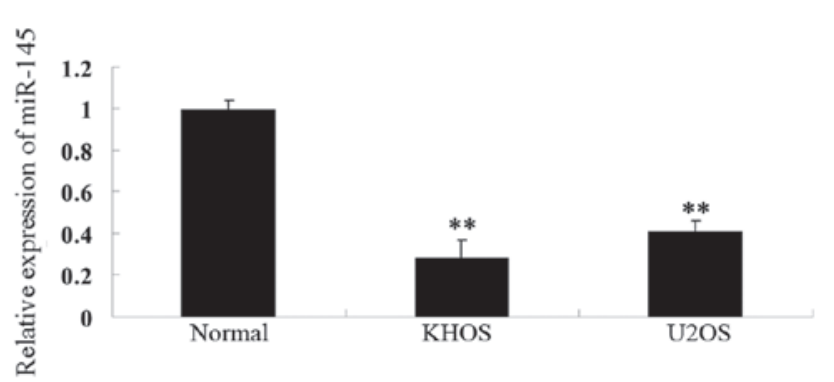

Figure 1. Downregulation of miR-145 in OS tissues and cell lines. (A) qPCR was performed to determine the miR-145 level in 18 OS tissues and their matched adjacent normal tissues. It was demonstrated that the miR-145 level was significantly downregulated in the OS tissues compared with their matched non-cancerous bone tissues. (B) qPCR was performed to determine the miR-145 level in normal bone tissues and two OS cell lines, KHOS and U2OS. ${ }^{* *} \mathrm{P}<0.01$ vs. normal bone tissues. OS, osteosarcoma; miR/miRNA, microRNA; qPCR, quantitative PCR; T, tumor tissue; N, normal tissue.

serum free medium. For the invasion assay, $500 \mu \mathrm{l}$ RPMI-1640 containing $10 \%$ FBS was added into the lower chamber. Next, $300 \mu 1$ of the cell suspension was added into the upper chamber. Following incubation for $24 \mathrm{~h}$, a cotton-tipped swab was used to gently remove non-invading cells and the ECMatrix gel from the interior of the inserts. A total of $500 \mu \mathrm{l}$ of staining solution was added to the unoccupied wells of the plate. Invasive cells on the lower surface of the membrane were stained by dipping inserts in the staining solution for $20 \mathrm{~min}$, and then rinsed with water and dried in the air. The cells were counted by capturing images of the membrane through a microscope.

Statistical analysis. SPSS 19.0 was used to perform the statis tical analysis (IBM, Armonk, NY, USA). All data are presented as the mean \pm standard deviation of at least three samples. One-way analysis of variance or Student's t-test was applied to perform the statistical analysis. $\mathrm{P}<0.05$ was considered to indicate a statistically significant difference.

\section{Results}

Expression of miR-145 is reduced in OS tissues and in KHOS and U2OS cells. qPCR was applied to examine the expression of miR-145 in the tissues of 18 cases of primary OS and their matched non-cancerous bone tissues. As demonstrated in Fig. 1A, the miR-145 level was significantly downregulated in the OS tissues compared with their matched non-cancerous bone tissues. Furthermore, the miR-145 expression in the OS cell lines, KHOS and U2OS, was investigated and found to be notably decreased compared with the expression in the normal bone tissues (Fig. 1B). Accordingly, these results indicate that miR-145 may have a suppressive role in the development of OS.

ROCK1 is a target gene of miR-145. Next, a bioinformatical analysis was performed to investigate the targets of miR-145, which may be crucial in OS. Several predicative software, including TargetScan (http://www.targetscan.org/), demonstrated that ROCK1 is a potential target based on putative target sequences at the 3'UTR position of ROCK1 (Fig. 2A). Since ROCK1 has been demonstrated to be involved in OS cell proliferation, migration and invasion, it was hypothesized that ROCK1 may also be involved in miR-145-mediated biological processes in OS cells. To investigate this hypothesis, the expression of ROCK 1 was examined in OS tissues and KHOS and U2OS cells. As demonstrated in Fig. 2B, the mRNA expression of ROCK1 was significantly increased in the OS tissues compared with their matched non-cancerous bone tissues. Furthermore, the protein level of ROCK1 was also upregulated in the KHOS and U2OS cells compared with the normal bone tissues (Fig. 2C). To further reveal the suppressive effect of miR-145 on ROCK1 expression, an miR-145 mimic was transfected into the KHOS and U2OS cells, and the mRNA and protein levels of ROCK1 were examined. As demonstrated in Fig. 2D, miR-145 inhibited the ROCK1 the protein level compared with the control and negative control groups. However, it had no effect on ROCK1 mRNA expression, which indicated that miR-145 has an inhibitory role in ROCK1 expression at a post-transcriptional level. Therefore, a luciferase activity assay was performed to confirm this. As demonstrated in Fig. 2E, co-transfection of 293T cells (ATCC) with miR-145 and wild-type ROCK1 3'-UTR led to a marked decrease in the luciferase activity, while co-transfection with miR-145 and mutant ROCK1 3'-UTR had no such effect. Therefore, ROCK1 was identified as a target gene of miR-145.

ROCK1 is involved in the miR-145-induced inhibition of cell proliferation of KHOS and U2OS cells. To further study the roles of miR-145 and ROCK1 in the OS cells, a cell proliferation assay was performed to determine the effects of miR-145 and ROCK1 overexpression on KHOS and U2OS cell proliferation. As demonstrated in Fig. 3A, co-transfection with miR-145 and ROCK1 attenuated the suppressive effect of miR-145 on ROCK1 protein expression. Furthermore, the cell proliferation assay data revealed that miR-145 significantly inhibited KHOS and U2OS cell proliferation compared with the controls. However, ROCK1 overexpression effectively reversed the miR-145-induced suppression of KHOS and U2OS cell proliferation (Fig. 3B). These data indicate that miR-145 inhibited OS cell proliferation, at least in part by downregulating ROCK1 protein expression.

ROCK1 overexpression reverses the inhibitory effects of miR-145 on KHOS and U2OS cell invasion. Next, the roles of miR-145 and ROCK1 in OS cell invasion were investigated. The results revealed that miR-145 significantly inhibited KHOS and U2OS cell invasion compared with the control groups. However, ROCK1 attenuated the suppressive effect of 
A

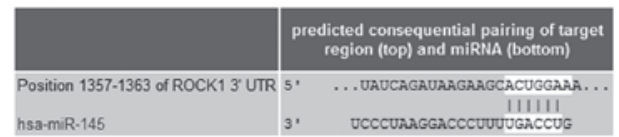

B

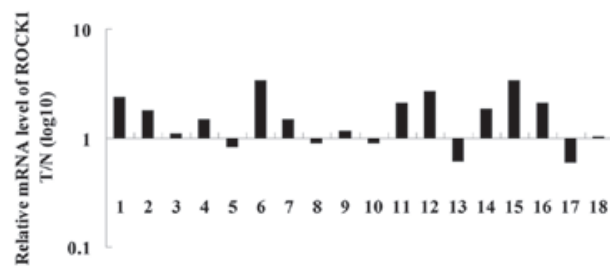

C

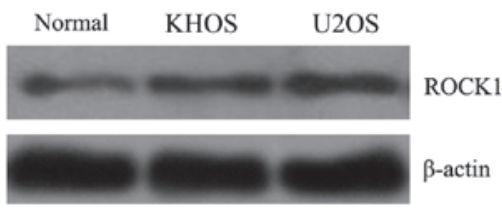

D

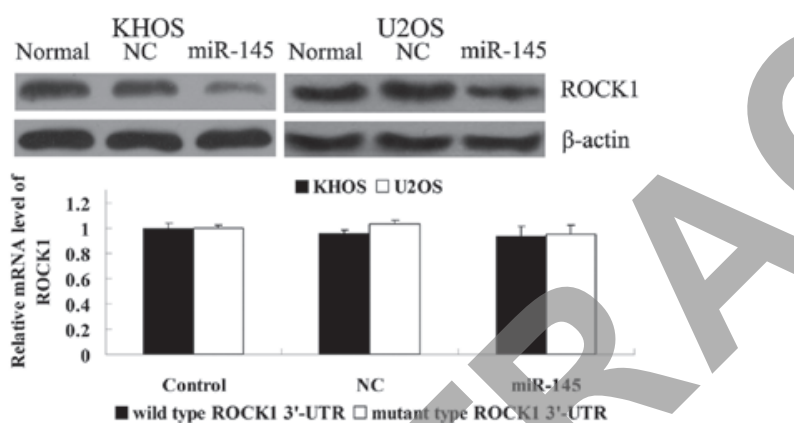

$\mathbf{E}$

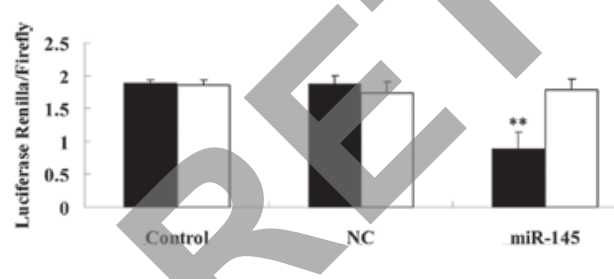

Figure 2. ROCK1 is a direct target gene of miR-145. (A) Data from TargetScan demonstrating the putative target sequence of miR-145 in 3'-UTR of ROCK1. (B) qPCR was performed to determine the ROCK1 mRNA level in 18 OS tissues and their matching adjacent normal tissues, and demonstrated that the level was significantly increased in the OS tissues compared with their matched non-cancerous bone tissues. (C) Western blotting was performed to determine the ROCK1 protein expression level in the normal bone tissues and the two OS cell lines, KHOS and U2OS, and demonstrated that the level was increased in KHOS and U2OS compared with the normal bone tissues. (D) Western blot analysis data revealing that miR-145 significantly downregulated the protein expression of ROCK1 in the KHOS and U2OS cells. However, miR-145 had no effect on the mRNA expression of ROCK1 in the KHOS and U2OS cells. Control, cells without transfection; NC, cells tranfected with negative control miRNA. (E) Luciferase report assay data demonstrating that co-transfection of 293T cells with miR-145 and wild-type ROCK1 3'-UTR led to a marked decrease in luciferase activity. However, co-transfection with miR-145 and mutant ROCK1 3'-UTR had no effect on luciferase activity, and co-transfection with the NC miRNA and wild-type ROCK1 3'-UTR or mutant ROCK1 3'-UTR also demonstrated no difference. Control, $293 \mathrm{~T}$ cells co-transfected with the blank vector and wild type ROCK1 3'-UTR or mutant ROCK1 3'-UTR. ${ }^{* *} \mathrm{P}<0.01$ vs. each other group. ROCK1, rho-associated protein kinase 1; miR/miRNA, microRNA; OS, osteosarcoma; qPCR, quantitative PCR; T, tumor tissue; $\mathrm{N}$, normal tissue; NC, negative control; 3'-UTR, 3' untranslated region.
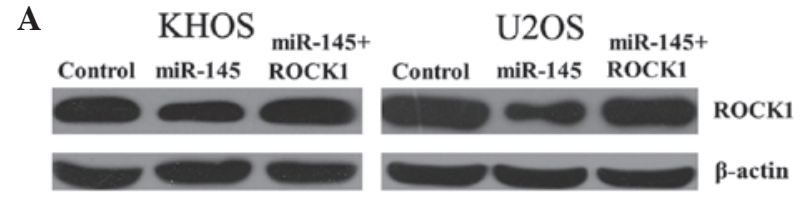

B

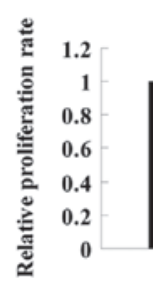

घHOS $\square$ U2OS
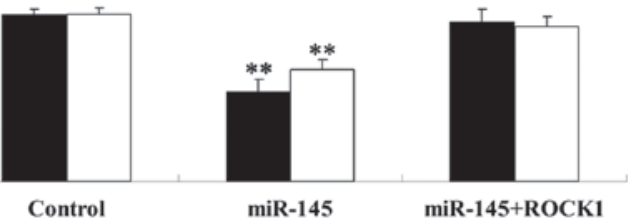

Figure 3. ROCK1 is involved in the miR-145-induced inhibition of cell proliferation of KHOS and U2OS cells. (A) Western blotting data demonstrating that co-transfection with miR-145 and ROCK1 attenuated the suppressive effect of miR-145 on ROCK1 protein expression. (B) Cell proliferation assay data revealing that miR-145 significantly inhibited KHOS and U2OS cell proliferation. However, ROCK1 overexpression effectively reversed the miR-145-induced suppression of cell proliferation. ${ }^{* *} \mathrm{P}<0.01$ vs. the control. Control, cells without any transfection; ROCK1, rho-associated protein kinase $1 ;$ miR/miRNA, microRNA.

miR-145 on KHOS and U2OS cell invasion (Fig. 4A). Since matrix metalloproteinase (MMP)2 and MMP9 have been indicated as being crucial in OS cell invasion, the protein expression of MMP2 and MMP9 in the KHOS and U2OS cells transfected with miR-145 mimic or co-transfected with miR-145 and ROCK1, respectively, was investigated. As demonstrated in Fig. 4B, consistent with the invasion assay data, miR-145 significantly inhibited the protein expression of MMP2 and MMP9 compared with the controls. However, ROCK1 overexpression reversed the miR-145-induced suppression of MMP2 and MMP9 protein expression in the KHOS and U2OS cells. These results indicate that miR-145 downregulated OS cell invasion, at least in part by inhibiting the protein expression of ROCK1.

\section{Discussion}

To the best of our knowledge, the present study reveals for the first time the regulatory mechanism of miR-145 and ROCK1 in the proliferation and invasion in OS cells. It was identified that miR-145 was frequently downregulated in the OS tissues and in the two cell lines, KHOS and U2OS. Furthermore, ROCK1 was identified as a novel target of miR-145 and it was demonstrated that the expression of ROCK1 was frequently reduced in OS tissues and cell lines. Furthermore, miR-145 significantly suppressed the protein expression of ROCK1 in the KHOS and U2OS cells. Following this, it was demonstrated that miR-145 overexpression suppressed OS cell proliferation and invasion, which was abrogated by the upregulation of ROCK1. Accordingly, these results indicate that miR-145 acts as a tumor-suppressor, at least in part by directly targeting ROCK1.

In fact, accumulating evidence has demonstrated that miR-145 has a suppressive role in multiple types of cancer, including lung, prostate, breast, glioma, gastric, bladder, ovarian and colon cancer, and in OS (11-17). However, data on the precise role of miR-145 in OS is limited. Recently, Fan et al 
A
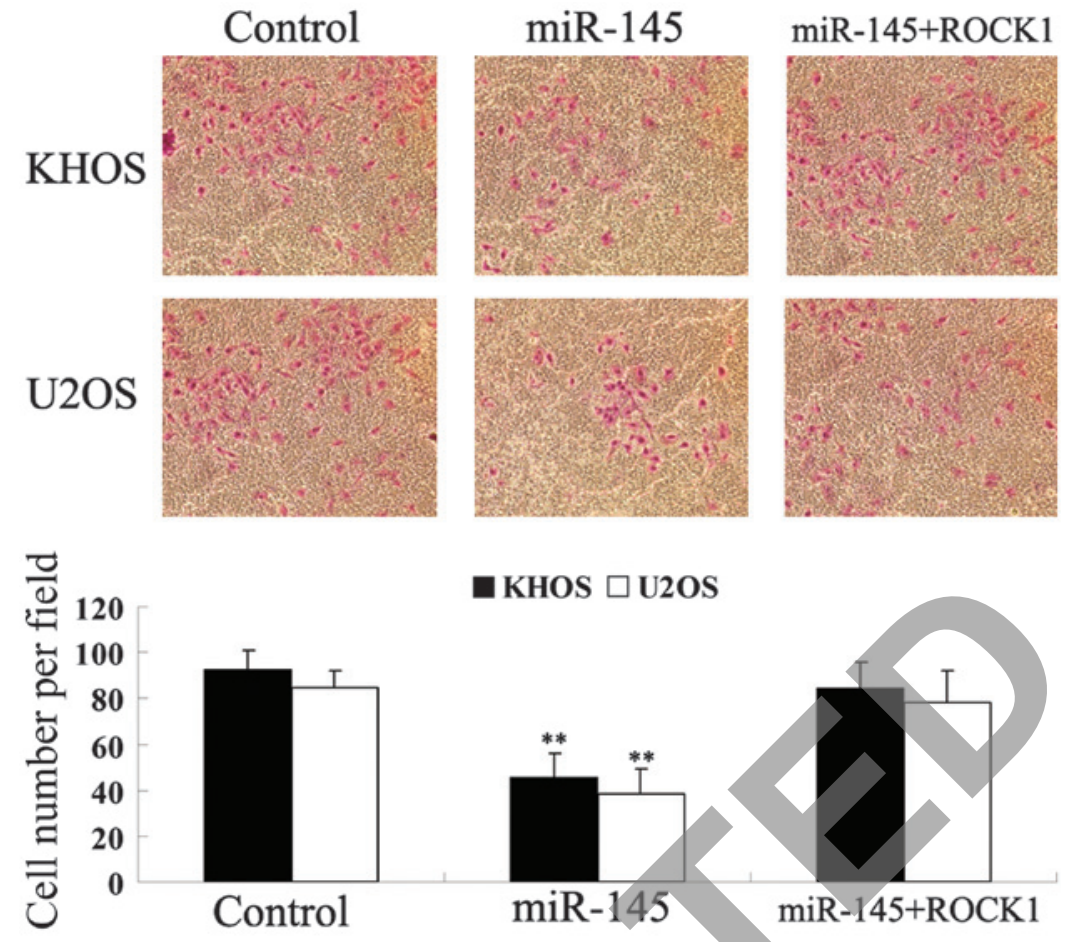

— KHOS $\square \mathrm{U} 2 \mathrm{OS}$

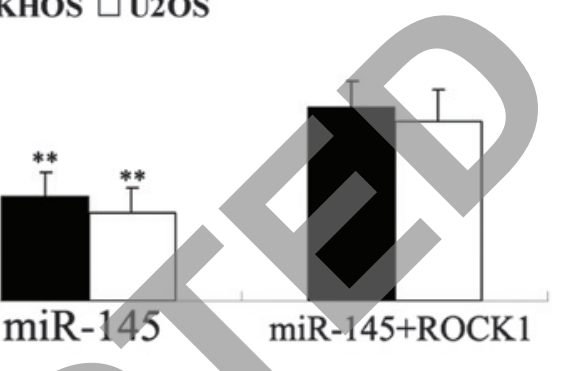

B

Figure 4. ROCK1 is involved in the miR-145-induced inhibition of OS cell invasion. (A) Cell invasion assay data demonstrating that miR-145 significantly inhibited KHOS and U2OS cell invasion. However, ROCK1 overexpression effectively reversed the miR-145-induced suppression of cell invasion. Control, cells without transfection. ${ }^{* *} \mathrm{P}<0.01$ vs. the control. (B) Western blotting data revealing that miR-145 significantly inhibited the protein expression of MMP2 and MMP9 in KHOS and U2OS cell invasion, which was reversed by ROCK1 overexpression. Control, cells without transfection; ROCK1, rho-associated protein kinase 1; miR/miRNA, microRNA; OS, osteosarcoma; MMP2/9, matrix metalloproteinase 2/9.

demonstrated that the expression of miR-145 was significantly reduced in OS tissues, and that the overexpression of miR-145 inhibited invasion, which was consistent with the findings of the present study (22). The study further demonstrated that miR-145 inhibited the angiopoiesis of OS, the mechanism of which involves its inhibitory effect on VEGF expression. Tang et al also identified that miR-145 was frequently downregulated in OS tissues, and that the downregulation of miR-145 was associated with OS aggressiveness and metastasis (17). Furthermore, the study also identified that OS patients with low miR-145 expression had poorer overall and disease-free survival times, indicating that miR-145 may be an independent prognostic marker for OS patients (17). As the present study identified ROCK1 as a novel target of miR-145 and found that miR-145 inhibited OS cell proliferation and invasion partially by downregulating ROCK 1 , these results expand our understanding of the molecular mechanisms by which miR-145 is involved in the regulation of the biological properties of OS cells.
It has been well established that the reorganization of the actin cytoskeleton has effects on cellular proliferation, adhesion and migration $(23,24)$. ROCK1 may be activated by binding to the active guanosine triphosphate-bound form of Rho, further interacting with the actin cytoskeleton, and therefore has a key role in cytoskeletal reorganization (25). Recently, Liu et al demonstrated that ROCK1 was highly expressed in OS tissues, and its upregulation was correlated with the poor prognosis of patients with OS (22). Furthermore, the forced inhibition of ROCK1 by siRNA suppressed cellular proliferation and viability, while inducing the apoptosis of OS cells (22). To the best of our knowledge, the present study demonstrates for the first time that ROCK1 is involved in the miR-145-induced inhibition of proliferation and the invasion of OS cells. Zhou et al recently found similar results, in that miR-340 suppressed OS growth and metastasis by directly targeting ROCK1 (26). Based on this accumulative evidence, it is indicated that ROCK1 has a crucial role in the development and progression of OS. 
In conclusion, the present study demonstrated that miR-145 was downregulated in the OS tissues and cell lines, and that its ectopic expression suppressed cell proliferation and invasion, at least partly by directly inhibiting the protein expression of ROCK1, which was identified as a novel target of miR-145. Therefore, miR-145 and ROCK1 are indicated to be novel promising candidates for developing effective therapeutic strategies for the treatment of OS.

\section{Acknowledgements}

This study was supported by Hunan Provincial Innovation Foundation For Postgraduates (CX2014B072) and the Open-End Fund for the Valuable and Precision Instruments of Central South University (CSUZC2014046).

\section{References}

1. Yu X, Wu S, Wang X, Xu M, Xu S and Yuan Y: Late post-operative recurrent osteosarcoma: Three case reports with a review of the literature. Oncol Lett 6: 23-27, 2013.

2. Thompson LD: Osteosarcoma. Ear Nose Throat J 92: 288, 2013.

3. Yang $\mathbf{J}$ and Zhang W: New molecular insights into osteosarcoma targeted therapy. Curr Opin Oncol 25: 398-406, 2013.

4. Yates LA, Norbury CJ and Gilbert RJ: The long and short of microRNA. Cell 153: 516-519, 2013.

5. Shukla GC, Singh J and Barik S: MicroRNAs: processing, maturation, target recognition and regulatory functions. Mol Cell Pharmacol 3: 83-92, 2011.

6. Bienertova-Vasku J, Sana J and Slaby O: The role of microRNAs in mitochondria in cancer. Cancer Lett 336: 1-7, 2013.

7. Profumo V and Gandellini P: MicroRNAs: cobblestones on the road to cancer metastasis. Crit Rev Oncog 18: 341-355, 2013.

8. Duan Z, Choy E, Harmon D, et al: MicroRNA-199a-3p is downregulated in human osteosarcoma and regulates cell proliferation and migration. Mol Cancer Ther 10: 1337-1345, 2011.

9. Huang G, Nishimoto K, Zhou Z, Hughes D and Kleinerman ES: miR-20a encoded by the miR-17-92 cluster increases the metastatic potential of osteosarcoma cells by regulating Fas expression. Cancer Res 72: 908-916, 2012.

10. Osaki M, Takeshita F, Sugimoto Y, et al: MicroRNA-143 regulates human osteosarcoma metastasis by regulating matrix metalloprotease-13 expression. Mol Ther 19: 1123-1130, 2011.
11. Campayo M, Navarro A, Viñolas N, et al: Low miR-145 and high miR-367 are associated with unfavourable prognosis in resected nonsmall cell lung cancer. Eur Respir J 41: 1172-1178, 2013.

12. Speranza MC, Frattini V, Pisati F, et al: NEDD9, a novel target of miR-145, increases the invasiveness of glioblastoma. Oncotarget 3: 723-734, 2012.

13. Villadsen SB, Bramsen JB, Ostenfeld MS, et al: The miR-143/-145 cluster regulates plasminogen activator inhibitor-1 in bladder cancer. Br J Cancer 106: 366-374, 2012.

14. Chiyomaru T, Tatarano S, Kawakami K, et al: SWAP70, actin-binding protein, function as an oncogene targeting tumor-suppressive miR-145 in prostate cancer. Prostate: Feb 10, 2011 (Epub ahead of print).

15. Takagi T, Iio A, Nakagawa Y, Naoe T, Tanigawa N and Akao Y: Decreased expression of microRNA-143 and -145 in human gastric cancers. Oncology 77: 12-21, 2009.

16. Zhang W, Wang Q, Yu M, Wu N and Wang H: MicroRNA-145 function as a cell growth repressor by directly targeting c-Myc in human ovarian cancer. Technol Cancer Res Treat: Aug 2, 2013 (Epub ahead of print).

17. Tang M, Lin L, Cai H, Tang J and Zhou Z: MicroRNA-145 downregulation associates with advanced tumor progression and poor prognosis in patients suffering osteosarcoma. Onco Targets Ther 6: 833-838, 2013.

18. Surma M, Wei L and Shi J: Rho kinase as a therapeutic target in cardiovascular disease. Future Cardiol 7: 657-671, 2011.

19. Amano M, Nakayama M and Kaibuchi K: Rho-kinase/ROCK: A key regulator of the cytoskeleton and cell polarity. Cytoskeleton (Hoboken) 67: 545-554, 2010.

20. Li J, Wang J, Jiao H, Liao J and Xu X: Cytokinesis and cancer: Polo loves ROCK'n' Rho(A). J Genet Genomics 37: 159-172, 2010.

21. Liu X, Choy E, Hornicek FJ, et al: ROCK1 as a potential therapeutic target in osteosarcoma. J Orthop Res 29: 1259-1266, 2011

22. Fan L, Wu Q, Xing X, Wei Y and Shao Z: MicroRNA-145 targets vascular endothelial growth factor and inhibits invasion and metastasis of osteosarcoma cells. Acta Biochim Biophys Sin (Shanghai) 44: 407-414, 2012.

23. Chen RH, Wang WJ and Kuo JC: The tumor suppressor DAP-kinase links cell adhesion and cytoskeleton reorganization to cell death regulation. J Biomed Sci 13: 193-199, 2006.

24. Bécart S and Altman A: SWAP-70-like adapter of T cells: a novel Lck-regulated guanine nucleotide exchange factor coordinating actin cytoskeleton reorganization and $\mathrm{Ca}^{2+}$ signaling in $\mathrm{T}$ cells. Immunol Rev 232: 319-333, 2009.

25. Hall A: Rho family GTPases. Biochem Soc Trans 40: 1378-1382, 2012.

26. Zhou X, Wei M and Wang W: MicroRNA-340 suppresses osteosarcoma tumor growth and metastasis by directly targeting ROCK1. Biochem Biophys Res Commun 437: 653-658, 2013. 